\title{
EVALUACIÓN DE RIESGOS EN LOS SECTORES TURISMO Y VIVIENDA FRENTE AL CAMBIO CLIMÁTICO EN LA COSTA DEL PRINCIPADO DE ASTURIAS
}

\author{
Alexandra TOIMIL ${ }^{1}$, Iñigo J. LOSADA ${ }^{1}$, Pedro DÍAZ-SIMAL ${ }^{1}$ \\ 'Instituto de Hidráulica Ambiental "IHCantabria" (Universidad de Cantabria - Fundación IH) \\ Avda. Isabel Torres 15, Parque Científico y Tecnológico de Cantabria 39011, Santander, España. \\ toimila@unican.es, losadai@unican.com,diazp@unican.es
}

\section{RESUMEN}

Este trabajo presenta una metodología novedosa que permite analizar los impactos costeros de inundación y erosión por cambio climático, a escala regional y con una alta resolución. Para ello se han empleado modelos de impacto eficientes, calibrados con datos de campo. Se han utilizado bases de datos históricas y proyecciones de dinámicas marinas de alta resolución. Para una serie de escenarios que combinan cambios en las variables climáticas y en la exposición, se ha determinado el riesgo sobre los sistemas socioeconómico y natural en la costa del Principado de Asturias. Se muestran los costes de la inacción sobre los sectores vivienda y turismo agregados a nivel de concejo, y enfocados principalmente a la planificación territorial y a la elaboración de estrategias adaptación. turismo.

Palabras clave: cambio climático, inundación costera, erosión, riesgos, vivienda,

\begin{abstract}
This work presents a new methodology to analyze coastal impacts of flooding and erosion due to climate change, at regional level and with high resolution. To that end, efficient impact models have been employed and calibrated with field data. Highresolution historical databases and projections of marine dynamics have been used. For a number of scenarios that combine changes in climate variables and exposure, risk on the socioeconomic and natural systems has been estimated throughout the coast of Asturias. Costs of inaction on housing and tourism sectors are shown aggregated at municipal level, and mainly focusing on territorial planning and strategy building on adaptation.
\end{abstract}

Key words: climate change, coastal flooding, erosion, risks, housing, tourism.

\section{INTRODUCCIÓN}

El dinamismo y la alta vulnerabilidad que caracterizan a las áreas costeras de todo el mundo se traduce en una mayor exposición a una serie de peligrosidades entre las que se encuentra el aumento del nivel medio del mar, uno de los principales efectos del cambio climático. El cambio climático conlleva, además, posibles alteraciones en la excepcionalidad de eventos climáticos extremos, haciéndolos mucho más frecuentes de lo que hoy en día son. La combinación del aumento del nivel medio del 
mar y los cambios en los eventos extremos darán lugar a inundaciones especialmente acusadas en zonas bajas y acelerarán la erosión costera, un impacto particularmente preocupante en playas urbanas, donde no habría posibilidades de una retranqueo natural a partir del movimiento del sistema dunar.

La gran incertidumbre que existe en la evolución del riesgo hace necesaria la mejora del estado del conocimiento en materia de análisis de riesgos, desde el desarrollo y aplicación de nuevos procedimientos integrados hasta la elaboración de herramientas que permitan mejorar su evaluación. En este estudio se presenta una metodología para evaluar las consecuencias sobre la población, la vivienda, las infraestructuras críticas, la industria, la agricultura y los servicios ecosistémicos derivadas del impacto de inundación en la costa del Principado de Asturias (norte de España). Para ello, se han definido escenarios de riesgo que combinan proyecciones climáticas de eventos extremos y aumento del nivel medio del mar, y cambios en el sistema socioeconómico basados en tendencias históricas. Por otro lado, se propone una aproximación para evaluar las consecuencias que conlleva la erosión de playas sobre el turismo en términos de pérdida de valor de uso recreativo.

\section{MÉTODOS}

En este estudio se propone una metodología para analizar la inundación y la erosión costeras bajo distintos escenarios (Fig. 1), enmarcándose el análisis de estos impactos dentro de una metodología más general de riesgo. En este marco más general, el riesgo $(\mathrm{R})$ se define como la probabilidad de un evento y sus consecuencias negativas, como resultado de la combinación de peligrosidad $(\mathrm{P})$, definida a través de las dinámicas marinas, exposición (E), asociada al medio físico, y vulnerabilidad (V), ligada a las características socioeconómicas de la zona $(\mathrm{R}=\mathrm{P} \cdot \mathrm{E} \cdot \mathrm{V})$. De este modo, si alguno de estos factores aumenta, también lo hará el riesgo.

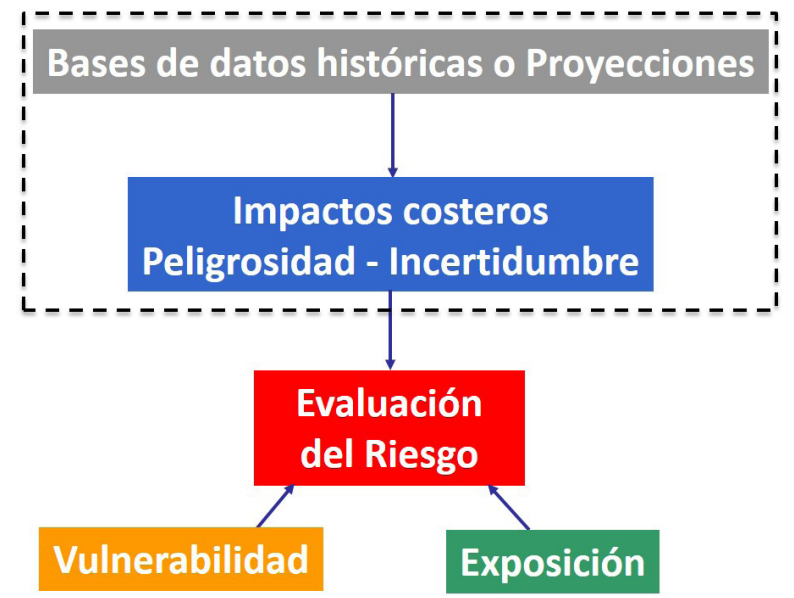

Fig. 1: Esquema de la metodología. 
El análisis del riesgo requiere, en primer lugar, la definición de la magnitud y duración de la peligrosidad, caracterizada en este caso a partir de las dinámicas marinas. Se ha contado con bases de datos históricas de alta resolución de oleaje en costa (DOW, Camus et al., 2013), marea meteorológica (GOS, Cid et al., 2014) y marea astronómica (reconstruida a partir de las componentes armónicas de la base de datos TPOX). Estas variables se combinan en un índice conocido como Cota de Inundación (CI), definido como la suma lineal de la MM, la MA y el Runup del oleaje $(\mathrm{Ru})$, éste último término calculado mediante la formulación de Stockdon et al. (2006). La reconstrucción histórica de las CI ha permitido reconstruir su régimen extremal y obtener cuantiles elevados de su distribución, principales responsables de los eventos de inundación. Se han estudiado las tendencias de los datos históricos y también se han realizado proyecciones locales de oleaje, marea meteorológica (Camus et al., 2014) y aumento del nivel medio del mar (Slangen et al., 2014) para los escenarios considerados, basados en Rutas Representativas de Concentraciones (RCP). Tras analizar los resultados obtenidos, se ha concluido que el nivel medio del mar es la única dinámica que sufrirá cambios significativos. Los años horizonte considerados son 2050 y 2100 , y los escenarios propuestos son: $+0.24 \mathrm{~m}$ a mitad de siglo y $+0.45 \mathrm{~m}$ (valor medio del RCP4.5 regionalizado en la costa asturiana), $+0.65 \mathrm{~m}$ (valor medio del RCP8.5 regionalizado en la costa asturiana) y $1 \mathrm{~m}$, a final de siglo. En el caso del impacto de inundación, dada la necesidad de considerar tanto la permanente como la debida a los eventos climáticos extremos, estos escenarios se han combinado con periodos de retorno de la CI de 100 y 500 años, de acuerdo con la Directiva Europea de Inundaciones.

La exposición y la vulnerabilidad, principales factores determinantes del riesgo, son dinámicas, varían en el tiempo y el espacio y dependen de factores económicos, sociales, geográficos, demográficos, culturales, institucionales y ambientales. La primera engloba las características físicas del área de estudio. Se ha trabajado con un Modelo Digital de Terreno (MDT) del Instituto Geográfico Nacional (IGN) basado en datos LIDAR de $5 \mathrm{~m}$ de resolución horizontal, en el que se han incluido las defensas costeras de puertos y encauzamientos, digitalizadas a partir de planos. La caracterización del tejido socioeconómico se ha llevado a cabo a través de un ráster de población de $100 \mathrm{~m}$ de resolución e indicadores económicos tales como el stock de capital y el Valor Añadido Bruto (VAB) sectorial desagregados a nivel de instalaciones y edificios en toda la franja costera, habiendo utilizado la distribución de renta per cápita como factor corrector del reparto. Además de esto, se han establecido parámetros clave en cada playa como el $\mathrm{D}_{50}$, y la altura de la berma. La vulnerabilidad, por otro lado, está relacionada con la susceptibilidad de los elementos expuestos a sufrir daños ocasionados por una amenaza concreta (peligrosidad) y depende del tipo de inundación que se produzca. Los posibles daños efectivos producidos por un evento extremo dependen de la cota de inundación alcanzada en cada zona y se determinan mediante la aplicación de curvas de vulnerabilidad; en el caso de inundación permanente la pérdida es total, independientemente del nivel de agua que se alcance. En este trabajo se han aplicado dos tipos de curvas de vulnerabilidad: 1) una función de daño que relaciona la CI con el porcentaje de daño efectivo para activos; y 2) una función de interrupción de negocio que relaciona esta misma $\mathrm{CI}$ con los días de pérdida de actividad para flujos económicos.

En lo que respecta a la exposición futura, se han empleado las proyecciones oficiales de población del Gobierno del Principado a mitad de siglo y, a final de 
siglo, se ha considerado como hipótesis razonable mantener la población actual. Las proyecciones de los indicadores económicos se han estimado en base al crecimiento tendencial mínimo observado en los últimos 30 años.

\section{RESULTADOS}

La evaluación de riesgos permite identificar las áreas más expuestas a diferentes impactos, entender el origen de los mismos, identificar su extensión y severidad, y asistir en la elaboración de planes de gestión y toma de decisiones tales como estrategias de adaptación.

\subsection{Inundación}

La inundación costera se ha caracterizado a alta resolución con un modelo hidrodinámico bidimensional cuya calibración se ha realizado a partir de datos de campo obtenidos por IHCantabria tras los temporales del invierno de 2014. Las dinámicas generadoras del impacto se han combinado en un indicador de nivel del mar total (o cota de inundación) que alimenta al modelo a través de una serie de puntos a lo largo de la costa (Fig. 2).

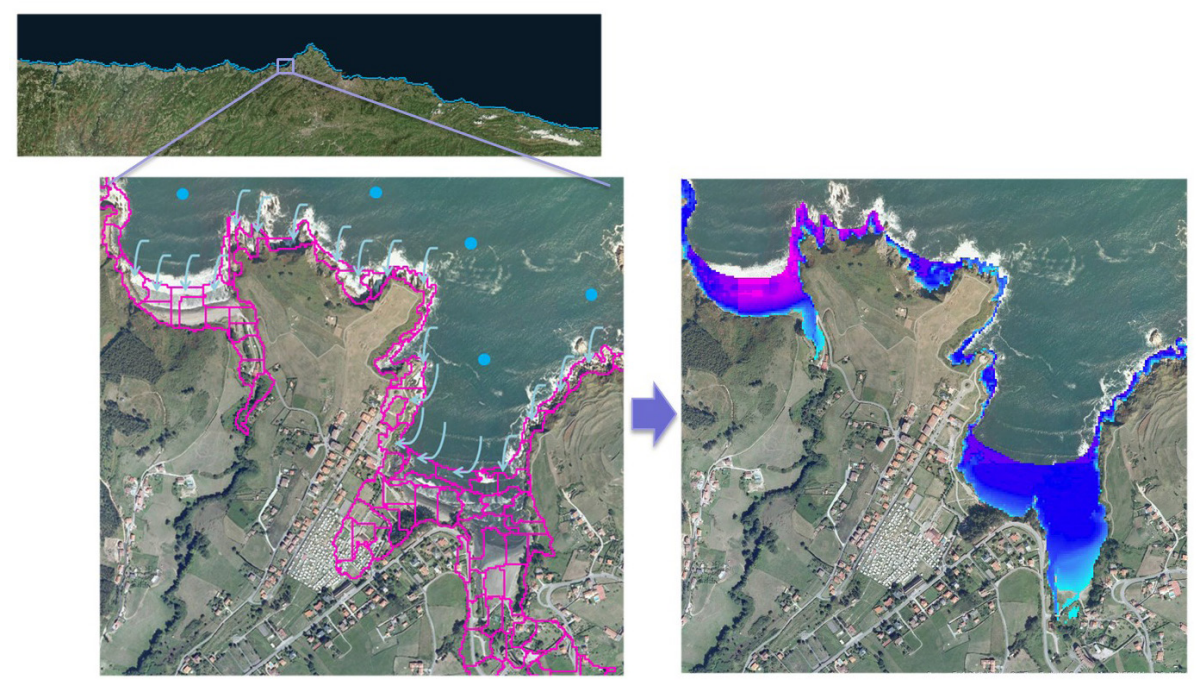

Fig. 2: Esquema conceptual del modelado de inundación. Fuente: elaboración propia

A la hora de evaluar las consecuencias económicas futuras es necesario poder comparar los resultados obtenidos para los distintos escenarios dentro del contexto socioeconómico presente, por ello se ha aplicado una tasa de descuento. Si bien existe una amplia gama de puntos de vista acerca del descuento, la mayoría coincide en diferenciar el medio y el largo plazo. En este trabajo hemos aplicado una tasa de descuento del $2 \%$ a 2050 y del $1 \%$ a 2100 . De este modo, es posible tener una idea de cuánto esfuerzo inversor resultaría hoy tolerable para resolver problemas futuros. No obstante, y dado que esto puede provocar cierta distorsión en lo que respecta a la importancia real de 
eventos que, pese a estar alejados en el tiempo conllevan graves consecuencias, se ha decidido presentar los resultados tanto descontados como sin descontar.

Tras cruzar los mapas de inundación con los datos de exposición, es decir, con las capas de información socioeconómica localizada espacialmente, se ha obtenido el daño máximo sobre la población, el stock de capital y el VAB sectorial. Dado que cada elemento expuesto tiene una susceptibilidad diferente frente a un mismo impacto, y puesto que sólo en el caso de inundación permanente el daño es del 100\%, sobre esos daños máximos se han aplicado las curvas de vulnerabilidad correspondientes, obteniéndose así el daño efectivo. Las consecuencias negativas sobre los sistemas socioeconómico y natural del litoral asturiano se muestran agregadas a nivel de concejo con el objetivo de mejorar la planificación territorial y facilitar la identificación de puntos críticos con vistas a la priorización de la adaptación. El mayor riesgo se concentra en los sectores residencial e industrial, viéndose especialmente afectadas las zonas situadas a orillas de las rías. Como ejemplo, la figura 3 muestra el daño efectivo sobre el continente de los activos residenciales de la costa del Principado de Asturias a lo largo del siglo XXI. Los resultados están expresados en porcentaje sobre el stock de capital residencial de toda la provincia, que asciende a 26 billones de euros (referido a 2011 en euros constantes de 2006, Fundación BBVA). Se puede observar que Ribadesella es el concejo susceptible de sufrir los mayores daños, que podrían ascender a final de siglo, para la inundación de 100 años de periodo de retorno y el nivel medio del mar $63.5 \mathrm{~cm}$ más elevado, al $0.3 \%$ del valor del stock de viviendas asturianas (cerca de 78 millones de euros, resultado sin descontar).

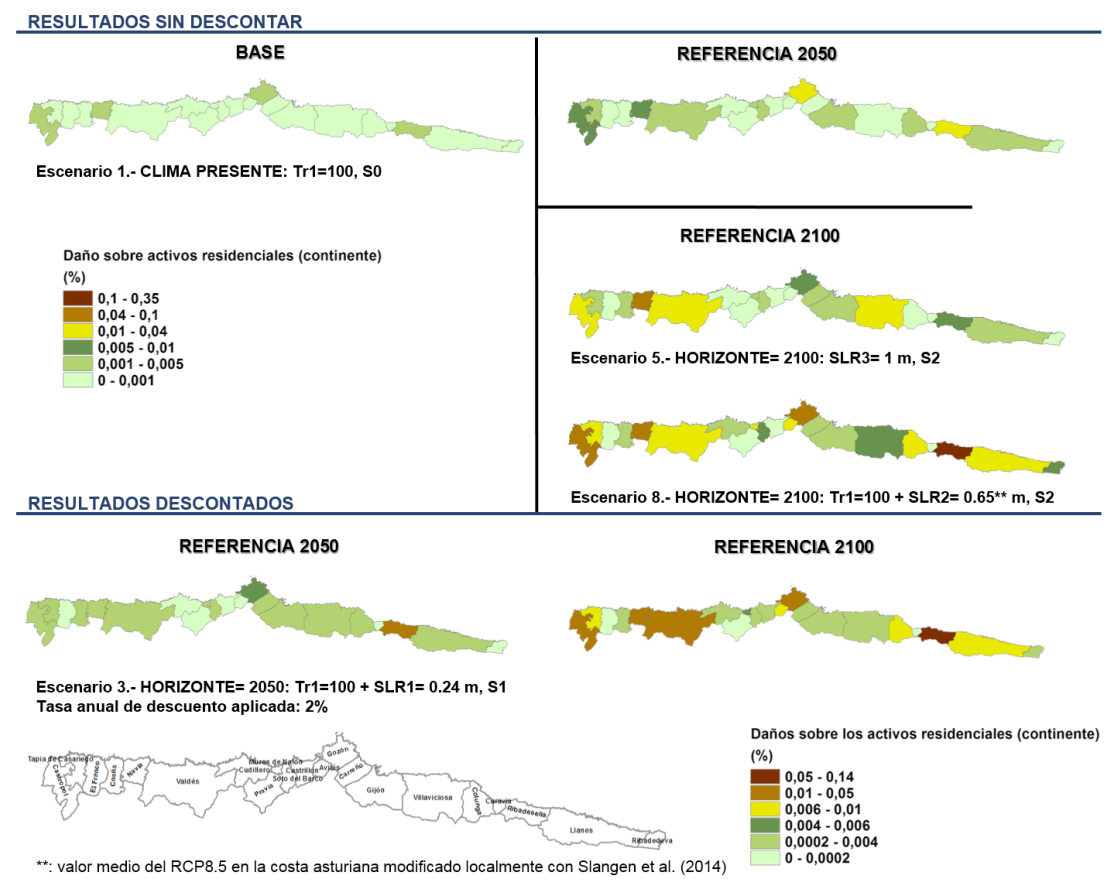

Fig. 3: Daño efectivo sobre los activos residenciales (continente) del litoral asturiano. 


\subsection{Erosión}

Para resolver la erosión costera, impacto que está poniendo en riesgo uno de los principales recursos de la región, el turismo del llamado "sol y playa", se ha optado por la aplicación de modelos de equilibrio basados en el perfil transversal de playa para reproducir la evolución estacional y decadal de la línea de costa, considerando que los procesos transversales son aquellos que gobiernan las variaciones estacionales e interanuales de la mayoría de los sistemas morfodinámicos del litoral del Principado de Asturias. Se han reconstruido las series históricas y futuras de erosión-acreción de cada una de las playas de estudio, lo que ha permitido analizar sus regímenes medio y extremal así como obtener estadísticos significativos. La figura 4 muestra, para un aumento de $1 \mathrm{~m}$ del nivel medio del mar (escenario a fin de siglo), el cuantil de retroceso de playa seca de 25 años de periodo de retorno y su indicador de erosión asociado. Este indicador se ha obtenido como el cociente entre el cuantil de retroceso y el ancho medio de cada playa, y nos da una idea del nivel de criticidad de cada una de ellas, ayudando a establecer prioridades de actuación. Se puede observar cómo muchas de las playas situadas al oeste del Cabo Peñas podrán llegar a sufrir retrocesos de hasta $25 \mathrm{~m}$ con relativa frecuencia.
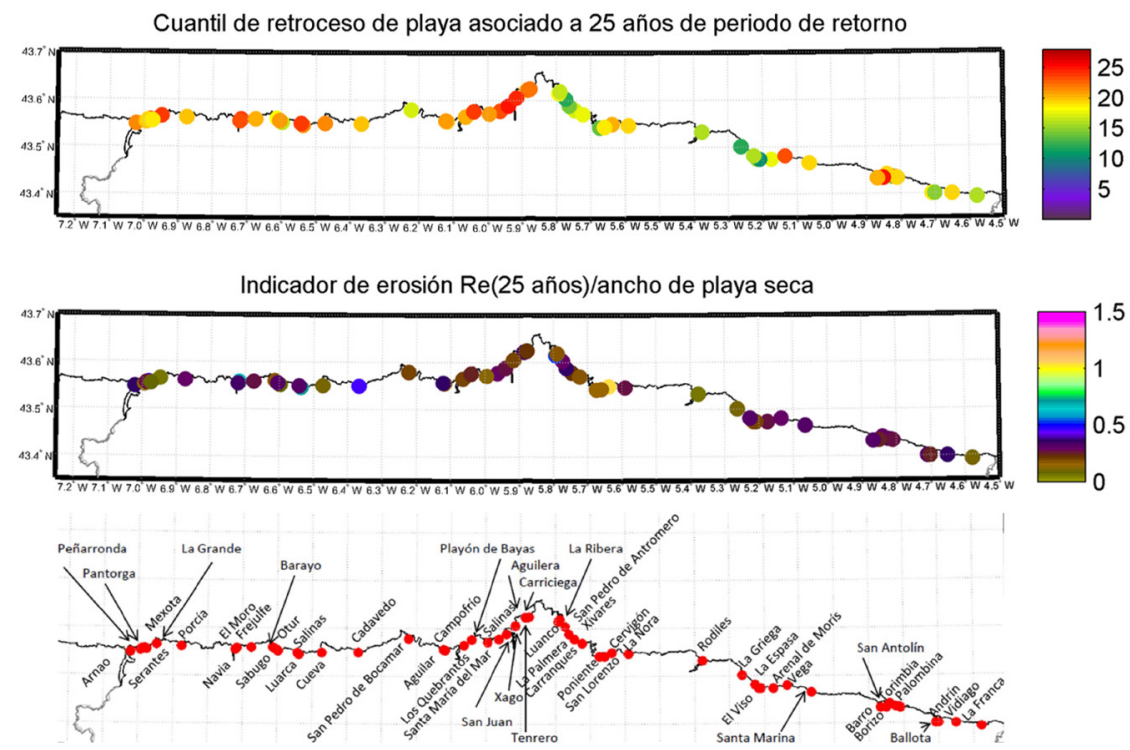

Fig. 4: Retroceso de 25 años de periodo de retorno en las playas de Asturias para un aumento del nivel medio del mar de $1 \mathrm{~m}$ (panel superior); indicador de erosión asociado al retroceso de 25 años de periodo de retorno (panel intermedio); localización de las playas de estudio (panel inferior).

Dada la gran variabilidad asociada al proceso de evaluación del valor recreativo de las playas se ha considerado razonable asumir la hipótesis de mantener constante la estructura salarial de la sociedad, las preferencias de los usuarios y los servicios complementarios que ofrecen a día de hoy las playas objeto de este estudio. Por sus 
características y en base al área potencialmente perdida por efecto del aumento del nivel medio del mar, las playas de Salinas y San Lorenzo podrán alcanzar pérdidas totales acumuladas de valor de uso recreativo de hasta 175 y 150 millones de euros, respectivamente, a final de siglo. La figura 5 muestra los resultados agregados a nivel de concejo. Bajo la hipótesis de un aumento del nivel medio del mar de $1 \mathrm{~m}$, Castrillón podrá alcanzar daños sobre el uso recreativo de sus playas por valor de más de 300 millones de euros, y Gijón, Llanes, Valdés y Gozón de hasta 200 millones de euros (agregados a final de siglo).
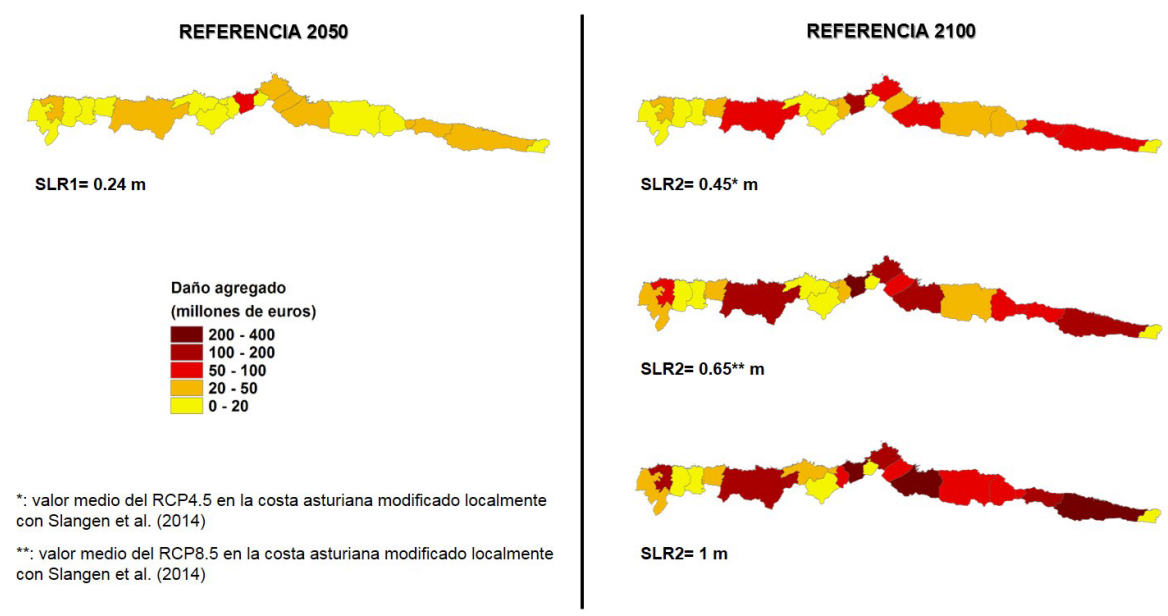

Fig. 5: Pérdida de valor contable de uso recreativo de las playas del Principado de Asturias.

\section{DISCUSIÓN}

La estimación del riesgo planteada en este trabajo supone un paso más en el estudio del cambio climático en la costa y sienta bases más sólidas para el planteamiento de estrategias y medidas de adaptación orientadas a sectores. Sin embargo, hay que tener en cuenta que existen ciertas limitaciones asociadas a los datos disponibles y a los modelos numéricos empleados, que deben tenerse en cuenta para interpretar de forma adecuada los resultados.

Este análisis ha permitido identificar las áreas geográficas más vulnerables, así como cuantificar la magnitud del impacto generado sobre las mismas. No obstante, es importante señalar que no se está realizando un pronóstico de daños sino más bien un ejercicio de ensayo en el que se presenta lo que podría observarse en el Principado de Asturias ante escenarios de riesgo. Este ensayo pretende ser representativo de posibles evoluciones de la sociedad afectada, mostrando la variación de las consecuencias que se podrían percibir para así poder mostrar cómo podrían contribuir las estrategias de adaptación con las que cuenta la sociedad y evaluar su posible aportación al bienestar social.

Los resultados presentados son, por tanto, indicadores de la susceptibilidad actual de la población y de los ecosistemas así como de las consecuencias a las que se podría 
llegar ante la inacción frente al cambio climático. No se ha tenido en cuenta la reubicación de asentamientos urbanos en riesgo, la migración de especies ni el aprendizaje y maduración de la sociedad.

\section{AGRADECIMIENTOS}

El trabajo ha sido financiado por el Ministerio de Agricultura, Alimentación y Medio Ambiente en el marco del proyecto "Asistencia técnica a la elaboración de un estudio sobre la adaptación al cambio climático de la costa del Principado de Asturias". Los autores del artículo agradecen, además de la financiación proporcionada, el apoyo técnico recibido por el Gobierno del Principado de Asturias.

\section{REFERENCIAS}

Camus P, Méndez FJ, Medina R, Tomas A, Izaguirre C (2013). High resolution Downscaled Ocean Waves (DOW) reanalysis in coastal areas. Coastal Engineering, doi: 10.1016/j.coastaleng.2012.09.002

Camus P, Menéndez M, Méndez FJ, Izaguirre C, Espejo A, Cánovas V, Pérez J, Rueda AC, Losada IJ, Medina R (2014). A weather type statistical downscaling framework for ocean wave climate. Journal of Geophysical research, doi: 10.1002/2014JC010141.

Cid A, Castanedo S, Abascal AJ, Menéndez M, Medina R (2014) A high resolution hindcast of the meteorological sea level component for Southern Europe: the GOS dataset. Climate Dynamics, doi: 10.1007/s00382-013-2041-0.

Fundación BBVA: http://www.fbbva.es/ (última consulta: octubre de 2015).

Slangen ABA, Carson M, Katsman CA, van de Wal RSW, Köhl A, Vermeersen LLA, Stammer D (2014). Modelling twenty-first century regional sea-level changes. Climatic Change, doi: 10.1007/s10584-014-1080-9.

Stockdon HF, Holman RA, Howd PA, Sallenger AH (2006) Empirical parameterization of setup, swash, and runup. Coastal Engineering, doi: 10.1016/j. coastaleng.2005.12.005 